\title{
PRONY'S METHOD USED FOR TESTING HARMONICS AND INTERHARMONICS IN ELECTRICAL POWER SYSTEMS
}

\author{
Jarosław Zygarlicki' ${ }^{1)}$, Janusz Mroczka ${ }^{2)}$ \\ 1) Opole University of Technology, Institute of Power Engineering, Prószkowska 76, 45-758 Opole, Poland \\ (凶j.zygarlicki@po.opole.pl,+48 77400 0547) \\ 2) Wroclaw University of Technology, Chair of Electronic and Photonic Metrology, Bolestawa Prusa 53/55, 50-317 Wroctaw, Poland \\ (janusz.mroczka@pwr.wroc.pl)
}

\begin{abstract}
The article presents an application of Prony's method with some known components in the analysis of electric power quality. Modifications of the Prony algorithm broaden the scope of method application. Modification of the filter of known components enables more accurate analysis of the parameters of unknown components and components with known or assumed frequencies. This article presents a comparison of the results of analyses conducted with the proposed algorithm for simulated and real signals and the results obtained by means of a commercial electric power quality testing device, operating in class A and using the Fourier transform. The proposed method enables to estimate the levels of the harmonic components, the frequency of the fundamental signal and real parameters of the interharmonic components, which are grouped and averaged in the contemporary monitoring equipment. Knowledge of the individual parameters of the interharmonics has considerable diagnostic importance while removing causes of incorrect operation affecting sensitive equipment in some electric power systems. Additionally, the algorithm is capable of analyzing exponentially damped components and finds its application in analysis of disturbances, for example, transient oscillations.
\end{abstract}

Keywords: Prony's method, signal processing, harmonics, measurements.

\section{Introduction}

In the analysis of electric power quality parameters, information about the frequencies of signals' harmonics can easily be obtained as a multiplication of the fundamental frequency, whereas the frequencies of other disturbances are difficult to determine.

It is relatively easy to get information about the frequencies of harmonics by measuring the frequency of the fundamental process with one of the popular methods [1-2] and multiplying that frequency. However, obtaining information about real frequencies of interharmonics or subharmonics is much more difficult [3-5], therefore determination of the content of interharmonics in power signals requires compromise solutions [6-7].

Skipping the determination of real frequencies of interharmonics causes errors in power systems measurements. The effect of spectral leakage occurs, which results in incorrect information about all parameters of the signal components [8]. The lack of information about real frequencies of the power signal components makes interpretation of electric power quality analysis difficult.

Application of the Fourier transform, in which the sampling frequency is not synchronized with the frequency of the measured signal brings even greater errors in determination of components of the electric power signal, due to discrepancies between the frequencies of harmonics and frequencies of real components. The situation is improved when the window 
of analysis is synchronised in practical solutions. However, synchronization with all signal components is not possible due to the occurrence of interharmonics.

Several publications on the Prony method try to solve the problem of determining all real components of an electric power signal. There are yet serious problems in the implementation of the full Prony method, connected with estimation of many components in applications aiming to test the quality of electric power [9-10]. Calculation of a high-order Prony model results in significant computational complexity.

The method proposed in this article is the original implementation of Prony's algorithm [11] with some known components. The authors introduced to the algorithm original modifications to eliminate known components, what improved the accuracy of estimation of Prony model's parameters. The implemented method was analyzed for the possibility of its application in testing the quality of electric power. The tests were conducted in a real measuring system and the results were compared with the results obtained from the device used for analysis of electric power quality, characterized by class A uncertainty.

\section{Description of the method}

\subsection{Prony's method with some known components - theoretical basis}

The Prony method represents the signal as a combination of exponential functions [11]:

$$
\hat{x}_{n}=\sum_{k=1}^{p} \underline{h}_{k} \underline{z}_{k}^{n-1},
$$

where $\underline{h}_{k}$ represents a time-independent parameter defined as:

$$
\underline{h}_{k}=A_{k} \exp \left(j \theta_{k}\right)
$$

and $\underline{z}_{k}$ represents a time-dependent parameter, defined as:

$$
\underline{z}_{k}=\exp \left(\left(\alpha_{k}+j 2 \pi f_{k}\right) T\right)
$$

for $n=1,2, \ldots, N$, where: $N$ - the length of signal, $p$ - the number of exponentials, $T$ - the sampling period, $A_{k}$ - the amplitude of the $k$ th complex exponentials, $\alpha_{k}-$ the damping factor in seconds ${ }^{-1}, f_{k}$ - the frequency of the $k$ th component in $\mathrm{Hz}$ and $\theta_{k}-$ the initial phase of the $k$ th sinusoid in radians.

The textbook [11] assumes such applications of Prony's method where some exponential models (poles, attenuation or component frequencies) can be known a priori, and the relations between the components of these models and the amplitude and phase are unknown.

According to [11], assuming that $q$ exponential components $\underline{z}_{1} \ldots \underline{z}_{q}$ are known, the characteristic polynomial describing the relations between known components can be expressed by the formula:

$$
\prod_{k=1}^{q}\left(\underline{z}-\underline{z}_{k}\right)=\sum_{k=0}^{q} c_{k} \underline{z}^{k},
$$

where $c_{k}, k=1,2, \ldots, q-$ is a vector of combined coefficients. The characteristic polynomial for the $p$ components, with $q$ partially known and $p-q$ unknown parameters can be written as:

$$
\sum_{m=0}^{p} a_{m} \underline{z}^{m}=\left(\sum_{k=0}^{q} c_{k} \underline{z}^{k}\right)\left(\sum_{i=0}^{p-q} \alpha_{i} \underline{z}^{i}\right),
$$


where $\left\{\alpha_{i}\right\}$ is a vector of combined coefficients for unknown components and $\alpha_{p-q}=1$, and the $a_{m}$ parameters are coefficients of this polynomial. Further modification according to [11] gives:

$$
\sum_{m=0}^{p-q} \alpha_{m} y_{n-m}=0
$$

for $p+1 \leq n \leq 2 p$, where $y_{n}$ is defined as:

$$
y_{n}=\sum_{k=0}^{q} c_{k} x_{n-k}
$$

and coefficients $c_{k}$ can be calculated from (4). Equation (7) can be understood as filtering the original time sequence $x_{n}$, resulting in a new sequence $y_{n}$.

\subsection{The Prony method with some known components - algorithm implementation}

With reference to what has been mentioned in Section 2.1, the Prony method in which some components are known, can be developed as described in Fig. 1.

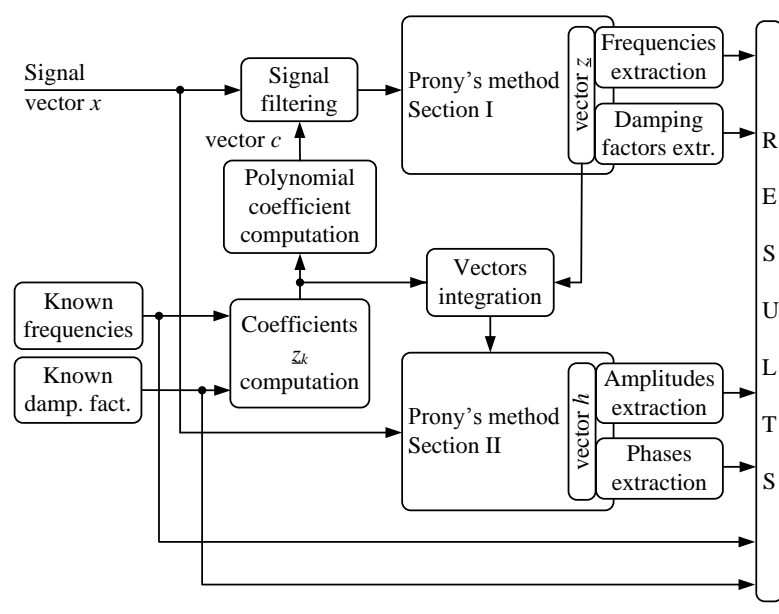

Fig. 1. Prony's method with some known components. Upper path: for interharmonics, lower path: for harmonics.

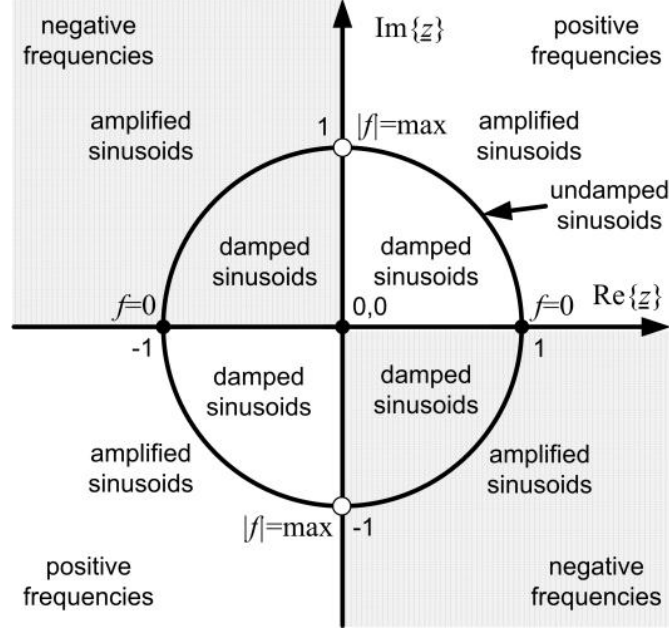

Fig. 2. Vector $\underline{z}$ and its importance in the representation of frequencies and exponential component attenuation coefficients in Prony's method.

The signal filter in a basic version of the algorithm ("signal filtering" block in Fig. 1) has a transfer function (8). Filtration occurs in a FIR-type filter (Finite Impulse Response) whose transmittance $H(\underline{z})$ can be presented by means of the dependence:

$$
H(\underline{z})=c_{0}+c_{1} \underline{z}^{-1}+\ldots+c_{L} \underline{z}^{-L} .
$$

The coefficients of the filter transmittance numerators $c_{k}$ can be calculated on the basis of dependence (1), where $\underline{z}_{k}$ make zeros of the filter transmittance.

It has been shown in the article [12] that on the assumption that the exponentials of the signal tested with Prony's method are not attenuated $\left(\alpha_{k}=0\right)$, the values of coefficients $z_{k}$ can be calculated on the basis of information about the frequencies of components in the signal:

$$
\operatorname{Re}\left\{\underline{z}_{k}\right\}=\frac{1}{\sqrt{1+\left(\tan \left(2 \pi T f_{k}\right)\right)^{2}}},
$$




$$
\operatorname{Im}\left\{\underline{z}_{k}\right\}=\frac{\tan \left(2 \pi T f_{k}\right)}{\sqrt{1+\left(\tan \left(2 \pi T f_{k}\right)\right)^{2}}} .
$$

The interpretation of vector $\underline{z}$ has been presented in Fig. 2. Zeros in the FIR filter transmittance in algorithm in Fig. 1 represent complex roots of the vector, calculated by means of (9) and (10). These zeros are located on a unitary circle in Fig. 2.

The vector of $c_{k}$ coefficients based on $\underline{z}_{k}$ roots is thus calculated analogically to the parameters $a_{m}$, when transformed from product of the equation to the sum in Prony's method [11].

The described process of calculating filter coefficients and the process of the filtration itself enables the preparation of the tested signal for further calculation of components with unknown parameters.

Elimination of known components by filtration permits reduction of the order in Prony's model at the next step - Section 1 of the full Prony least-squares method [12]. That improves the stability of final solutions and limits calculations in the Prony method by skipping the determination of component frequencies that had already been known or determined.

Section 2 of Prony method operates on the $\underline{z}_{k}$ coefficients vector built on the basis of the already known component frequencies and values determined in Section 1of Prony's method. Section 2 analyzes the original signal, prior to its filtering with an FIR filter.

Both Section 1 and Section 2 of the algorithm in Fig. 1 use extended calculations, based on the Prony least-squares method [12], which additionally improves the method's stability and accuracy.

The last stage of the algorithm in Fig. 1 consists in determining all parameters of components [12]. The problem of obtaining information about known frequencies in the algorithm in Fig. 1, treated as harmonic frequencies of the fundamental harmonic of the power signal, is solved by application of the algorithm of Fig. 3.

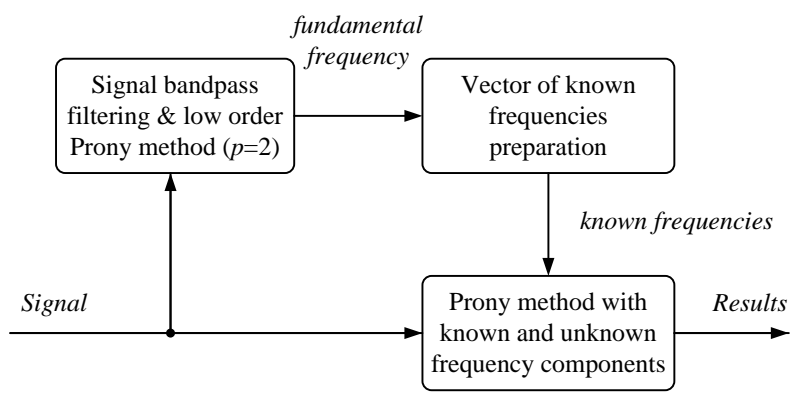

Fig. 3. Complete algorithm with determined frequencies of harmonics.

"Signal bandpass filtering \& low-order Prony method" block of the algorithm in Fig. 3 serves for determining the frequencies of the fundamental frequency, and has been presented in publication [1] together with the study. In the "Vector of known frequencies preparation" block, the vector of known frequencies is calculated according to:

$$
f=\left[\begin{array}{llll}
f_{h 0} & 2 f_{h 0} & \ldots & G f_{h 0}
\end{array}\right],
$$

where $G$ is the number of determined harmonics [1], $f_{h 0}$ is the fundamental frequency.

Two versions of the algorithm have been examined in the article:

- basic version - as described in Section 2,

- modified version - with improved accuracy. 
In the modified version of the algorithm the change refers to the filtration block of Fig. 1. The modification covers expansion of the scope of calculated values of coefficients $\underline{z}_{k}$ of vector $\underline{z}$ by the same values but having the real part negated, see Fig. 7.:

$$
\underline{z}:=\left[\begin{array}{ll}
\underline{z} & -\underline{z}^{*}
\end{array}\right]
$$

and accounting for them in the successive calculation steps in order to determine coefficients of the FIR filter. In effect, better attenuation of known components is achieved (the components to the right and left side of the plane $\underline{z}$ are attenuated - Fig. 2).

In both versions of the algorithm, when selecting the size of the Prony model, the highest possible order for unknown components has been adopted, in accordance with the computational capacity of the testing device. The tests reveal that increasing the model order leads to the improvement in the stability of the solutions and accuracy of calculations. This phenomenon has been presented in publication [13]. In the tests presented below the model order of $p=20$ turned out to be satisfactory. Further increase of the model order did not bring any improvement in the accuracy of the analysis.

\section{Measurement system and testing procedure}

Tests of methods were conducted for signals simulated in the system presented in Fig. 4a and for real signals - Fig. $4 \mathrm{~b}$.
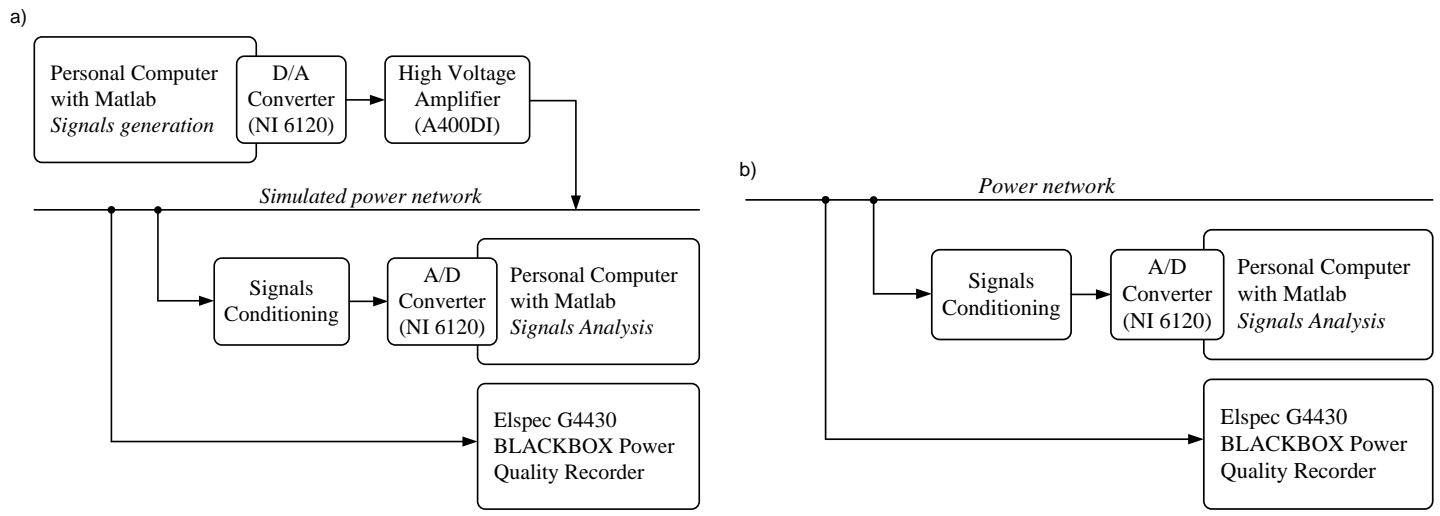

Fig. 4. System for testing the algorithm of the electric power quality analysis using Prony's method with some known components for: a) simulated signals; b) real signals.

The signal in the system presented in Fig. 4a is generated in the Matlab environment and sent to a NI-6120 card (National Instruments) with a digital-to-analogue converter. From the output of the converter, the test signal is fed to the high-voltage amplifier A400DI (FLC Electronics $\mathrm{AB}$ ) and then, from the input of the amplifier, it is fed to the test feeding line. The signal conditioning system equipped with the digital-to-analogue converter card is connected to that line. The digital form of the sampled signal is analyzed with the proposed algorithm that is implemented in the Matlab environment. Additionally, a commercially available analyzer of electric power quality G4430 (Elspec), operating with accuracy class A, is connected to the test feeding line [7].

Testing of that method was also performed for real-world signals. In the system presented in Fig. 4b, the G4430 analyzer and adjusted computer with the implemented algorithm were connected to a real power network.

At the first stage of tests carried out for the system in Fig. 4a, test signals were generated on the basis of the table of maximum amplitude values for individual harmonics for several initial harmonics according to [14]. 
Figures with fragments of tested voltage signals are shown in Fig. 5.
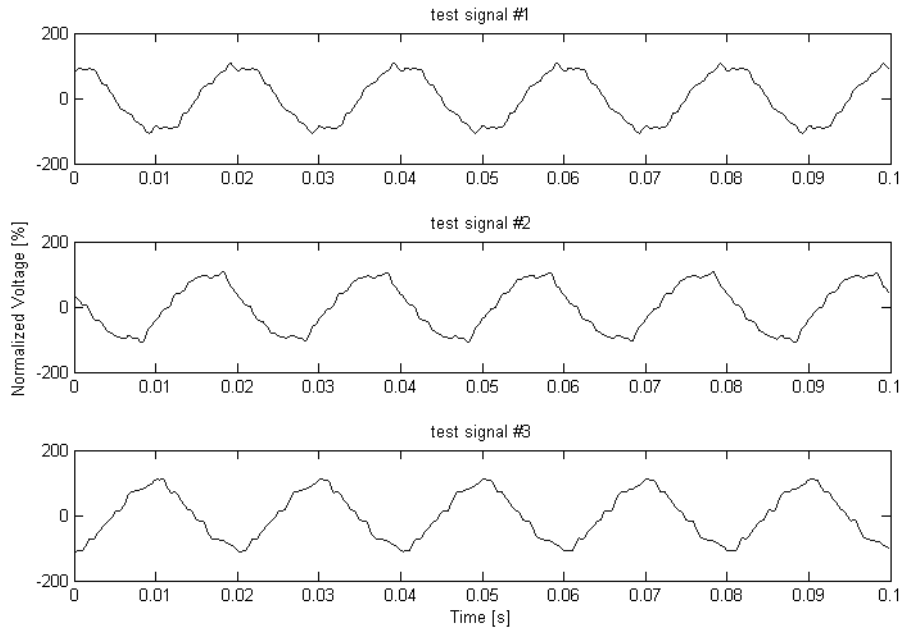

Fig. 5. The fragments of simulated test signals.

The signals should be as follows:

- test signal \#1 - apart from the component of the fundamental waveform, it should contain even harmonics with the parameters listed in Table 1;

Table 1. List of parameters of test signal \#1.

\begin{tabular}{|c|c|c|c|}
\hline No. & $\begin{array}{c}\text { Amplitude } \\
{[\%]}\end{array}$ & $\begin{array}{c}\text { Frequency } \\
{[\mathrm{Hz}]}\end{array}$ & $\begin{array}{c}\text { Initial phase } \\
{[\mathrm{deg} .]}\end{array}$ \\
\hline 1. & 100.00 & 50.00 & random \\
\hline 2. & 5.00 & 150.00 & random \\
\hline 3. & 6.00 & 250.00 & random \\
\hline 4. & 5.00 & 350.00 & random \\
\hline 5. & 1.50 & 450.00 & random \\
\hline 6. & 3.50 & 550.00 & random \\
\hline 7. & 3.00 & 650.00 & random \\
\hline 8. & 0.50 & 750.00 & random \\
\hline 9. & 2.00 & 850.00 & random \\
\hline 10. & 1.50 & 950.00 & random \\
\hline
\end{tabular}

Note: The amplitude is calculated in relation to the expected amplitude of the fundamental harmonic:

$\sqrt{2} \cdot 230 \mathrm{~V}=100 \%$.

- test signal \#2 - generated by adding an interharmonic component to the test signal \#1. The amplitude of the interharmonic equals $1 \%$ of the amplitude of the fundamental harmonic, random initial phase and frequency of $77 \mathrm{~Hz}$;

- test signal \#3 - generated by adding two interharmonic components to the test signal \#1. The amplitude of the interharmonic equals $1 \%$ of the amplitude of the fundamental harmonic, random initial phase and frequency $97 \mathrm{~Hz}$ and $695 \mathrm{~Hz}$.

Test signals were generated and measured in a real system (Fig. 4a), therefore adding more noise to the simulated test signals turned out to be unnecessary. The frequency of signal sampling was selected so be equal to $8 \mathrm{kHz}$ (at a resolution of $16 \mathrm{bits}$ ) as to meet the minimum sampling demands for Prony's method [12]. Test signals were used for comparison of the algorithms in basic and modified forms.

At the next stage of the study, real signals coming from a low-voltage power network (Fig. 4b) in normal operational conditions [14], recorded in the Opole University of Science and Technology, ul. Prószkowska 76, building II, were analyzed:

- real signal \#1 - date and time of acquisition: 2012.05.08, 12:59; 
- real signal \#2 - date and time of acquisition: 2012.05.09, 11:33;

- real signal \#3 - date and time of acquisition: 2012.05.09, 13:47.

The results of the analyses for the modified version of the algorithm were compared with the results obtained with the use of the Elspec G4430 device.

To evaluate the quality of the proposed methods of analysis, the parameters defining the maximum error of determination of amplitude $E_{A}$, maximum error of frequency $E_{F}$ determination and maximum error of signal reconstruction from determined parameters $E_{R}$ were used:

$$
E_{A}=\max \left|\frac{A-\hat{A}}{A}\right| \cdot 100 \%, \quad E_{F}=\max |F-\hat{F}|, \quad E_{R}=\max \left|\frac{x-\hat{x}}{x}\right| \cdot 100 \%,
$$

where: $A$ and $\widehat{A}$ are vectors of amplitudes of the set components and the components determined with the proposed methods, $F$ and $\hat{F}$ are vectors of the set and determined frequencies, $x$ and $\hat{x}$ represent the samples of set signal and the signal reconstructed on the basis of the determined parameters. Each experiment has been repeated 10 times for different values of the phase for simulated signals and the worst case (with the maximum value of parameter $E_{R}$ ) has been presented.

\section{Results}

Figs. 6-14 show the results of comparative tests for two proposed algorithms of analysis in basic and modified versions. Figs. 15-21 show simulation results obtained for real signals, in accordance with the diagram in Fig. 4b. The results obtained by applying the modified version of the method were compared with the results obtained by the Elspec G4430 device.
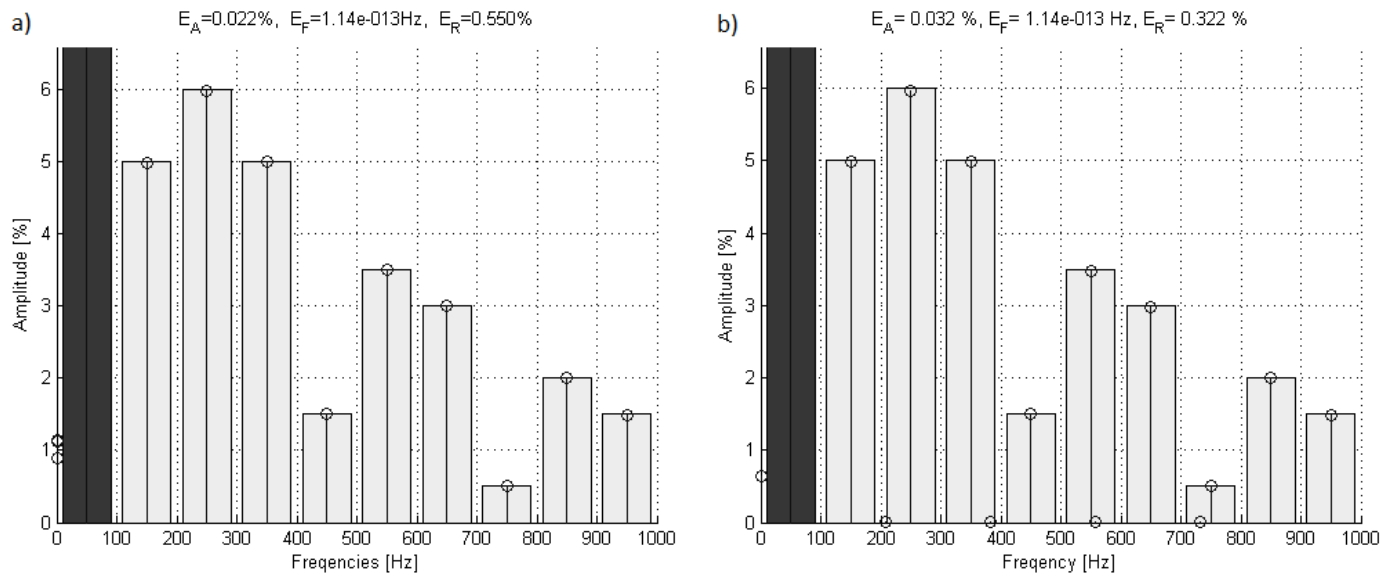

Fig. 6. Diagrams of set (bar graph) and determined (stem graph) amplitudes for test signal \#1, using: a) basic method; b) modified method.
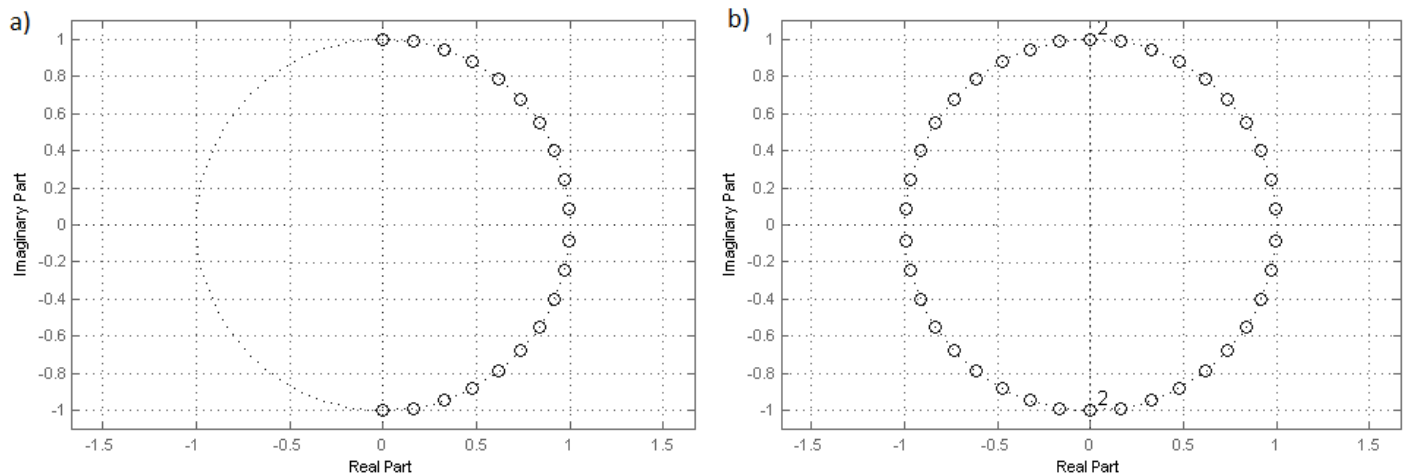

Fig. 7. Arrangement of zeros of the calculated FIR filter for signal \#1, for: a) basic method; b) modified method. 


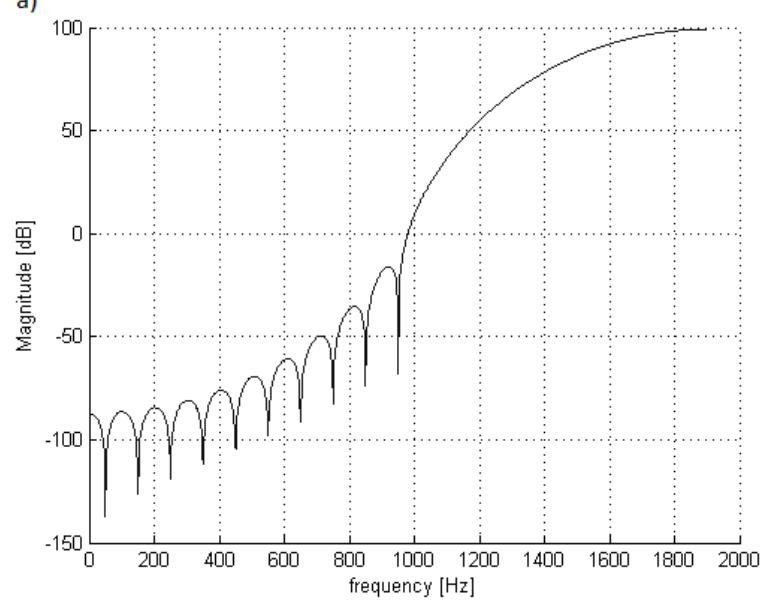

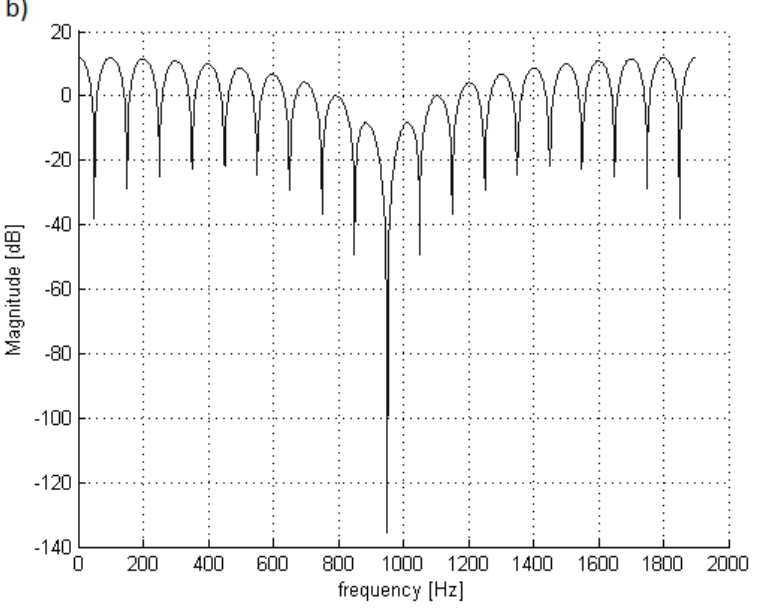

Fig. 8. Diagrams of the amplitude transient characteristics of the FIR filter for test signal \#1, for: a) basic method; b) modified method.

a)

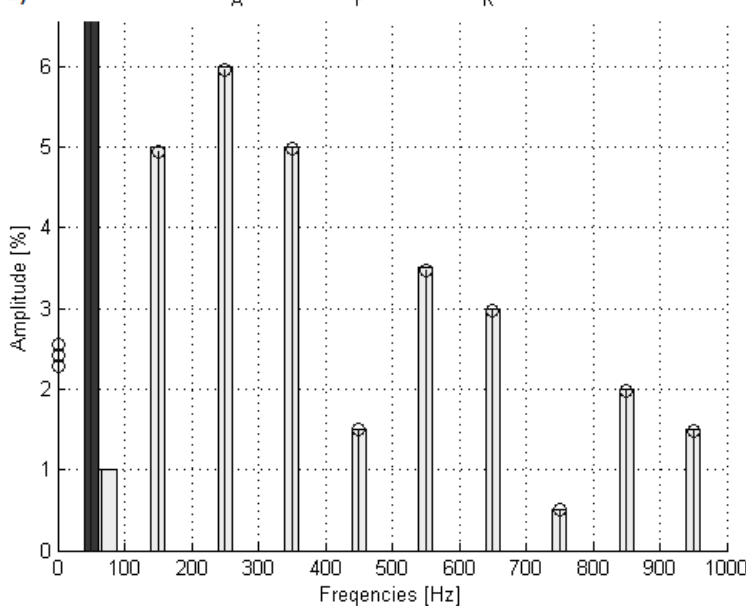

b) $\quad E_{A}=0.034 \%, E_{F}=37.2 m H z, E_{R}=0.434 \%$

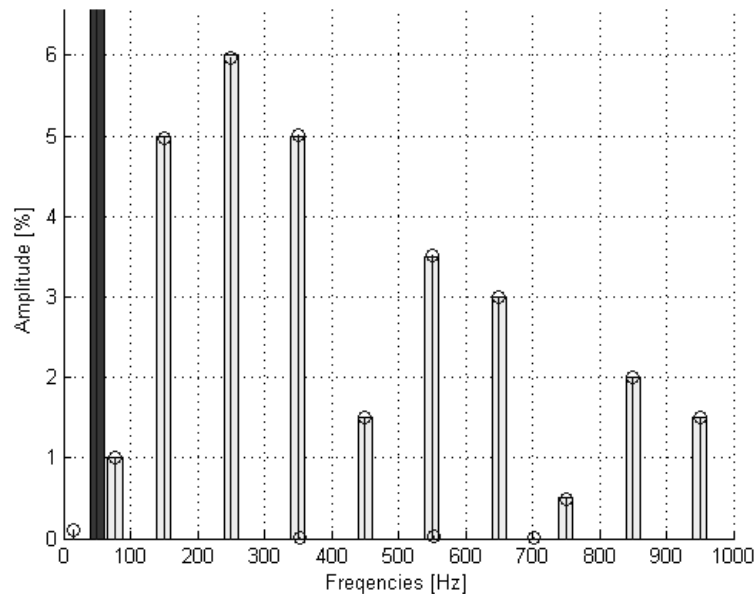

Fig. 9. Diagrams of set (bar graph) and determined (stem graph) amplitudes for test signal \#2, using: a) basic method; b) modified method.

a)

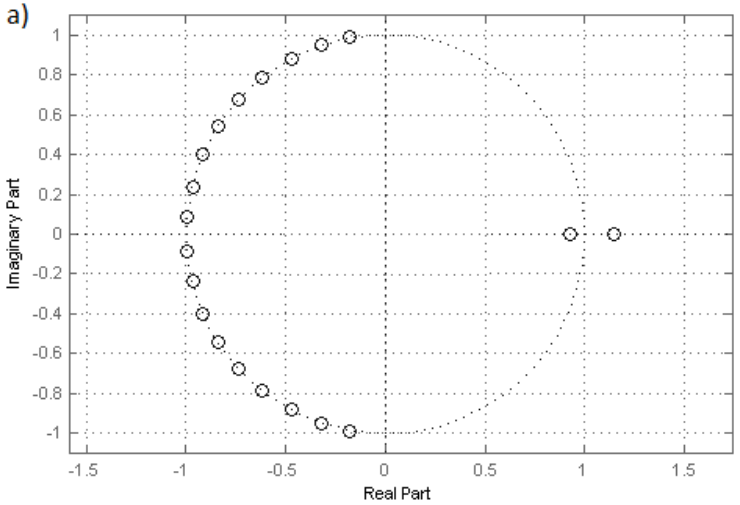

b)

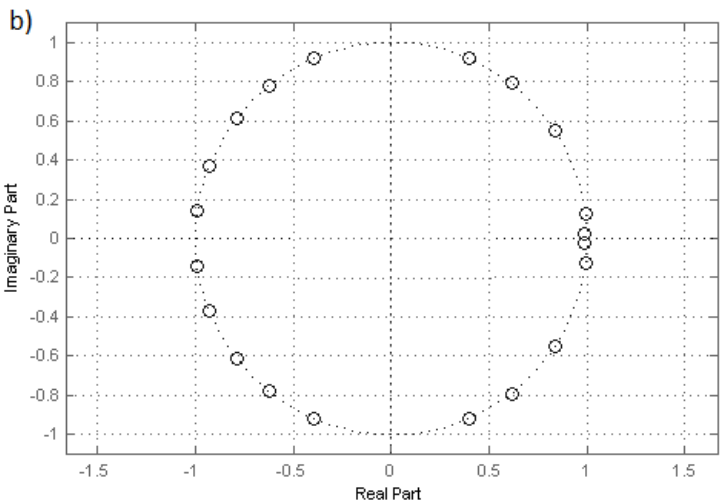

Fig. 10. Arrangement of coefficients $\underline{z}_{k}$ estimated in Section 1 of Prony's method for test signal \#2, for: a) basic method; b) modified method. 
a)

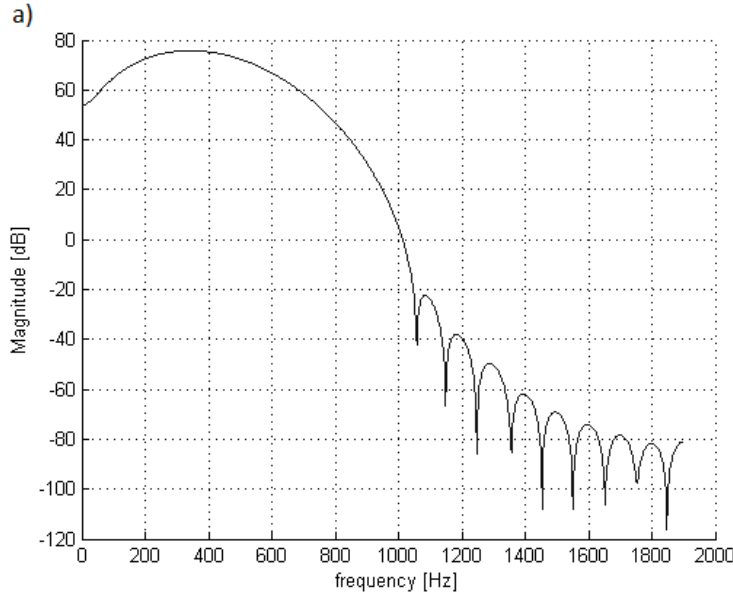

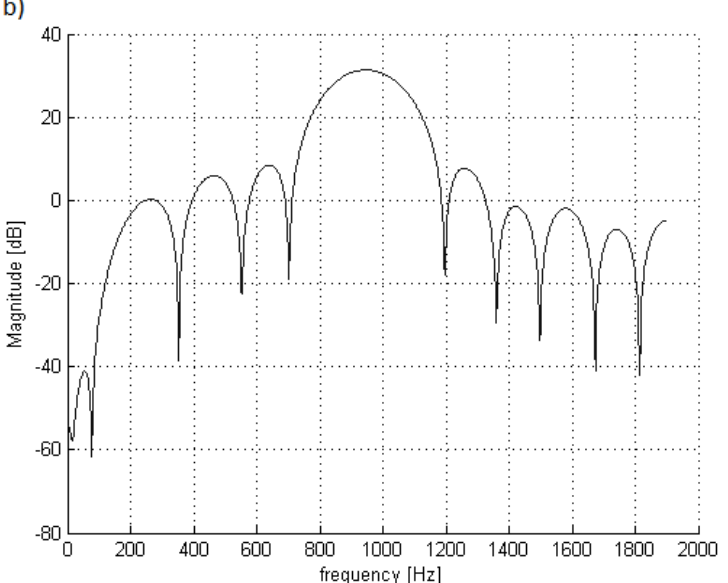

Fig. 11. Diagrams of the amplitude transient characteristics of the filter obtained from $z_{k}$ coefficients (Fig. 10), for: a) basic method; b) modified method.
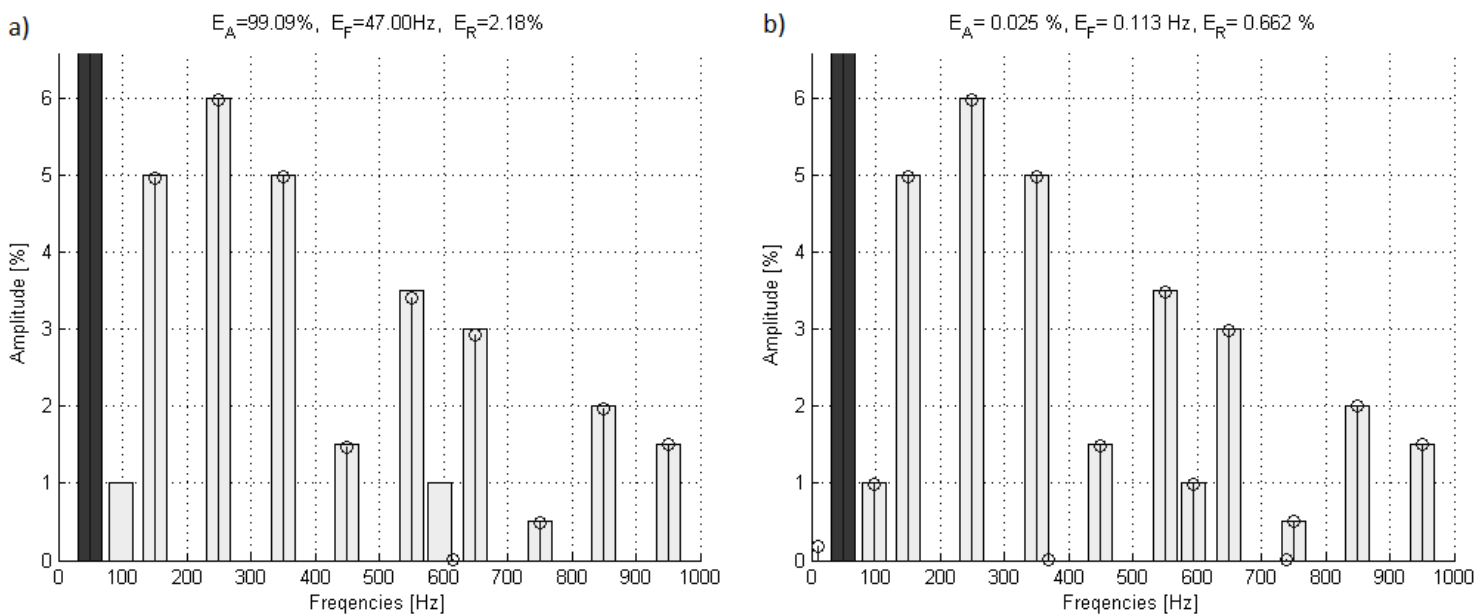

Fig. 12. Diagrams of set (bar graph) and determined (stem graph) amplitudes for test signal \#3, using: a) basic method; b) modified method.

a)

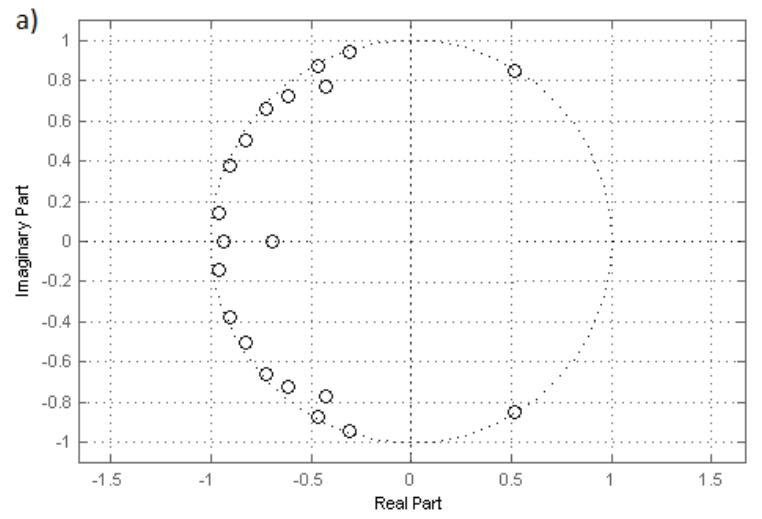

b)

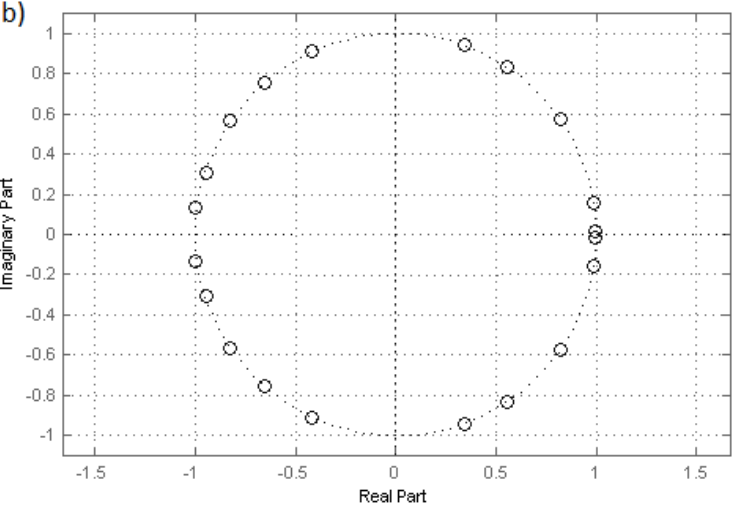

Fig. 13. Arrangement of coefficients $\underline{z}_{k}$ estimated in Section 1 of Prony's method for test signal \#3, for: a) basic method; b) modified method. 

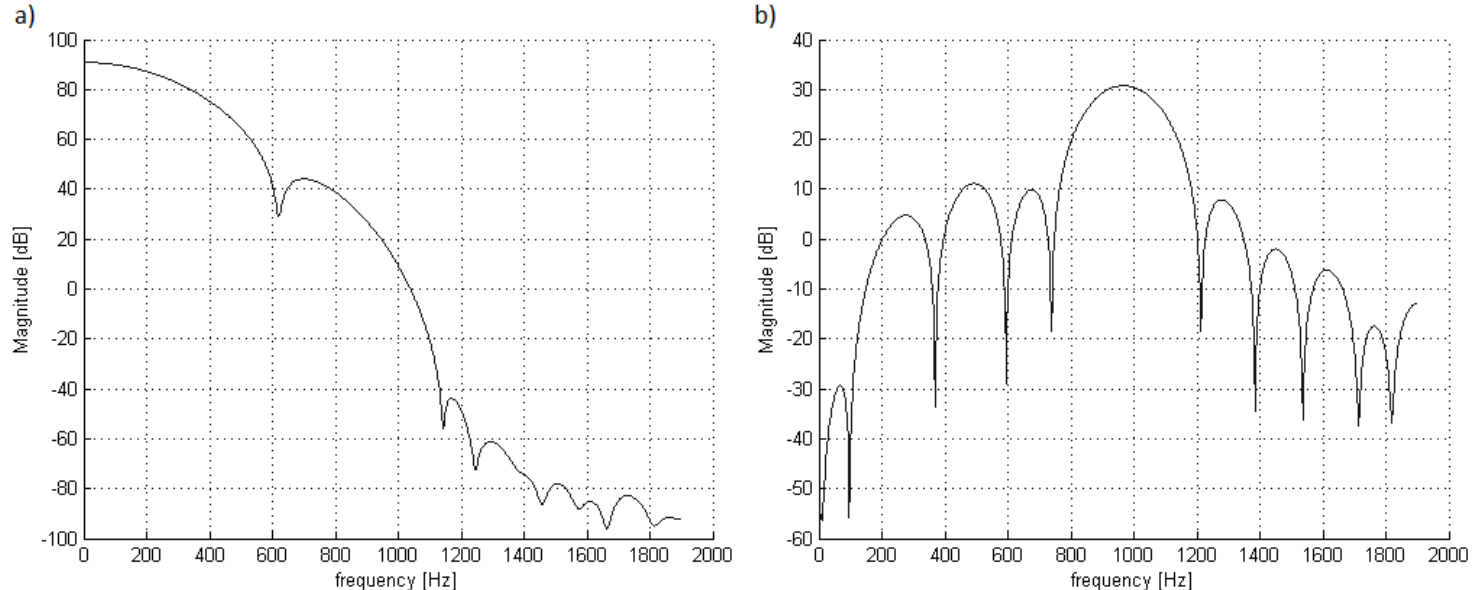

Fig. 14. Diagrams of the amplitude transient characteristics of the filter obtained from $z_{k}$ coefficients (Fig. 13), for: a) basic method; b) modified method.
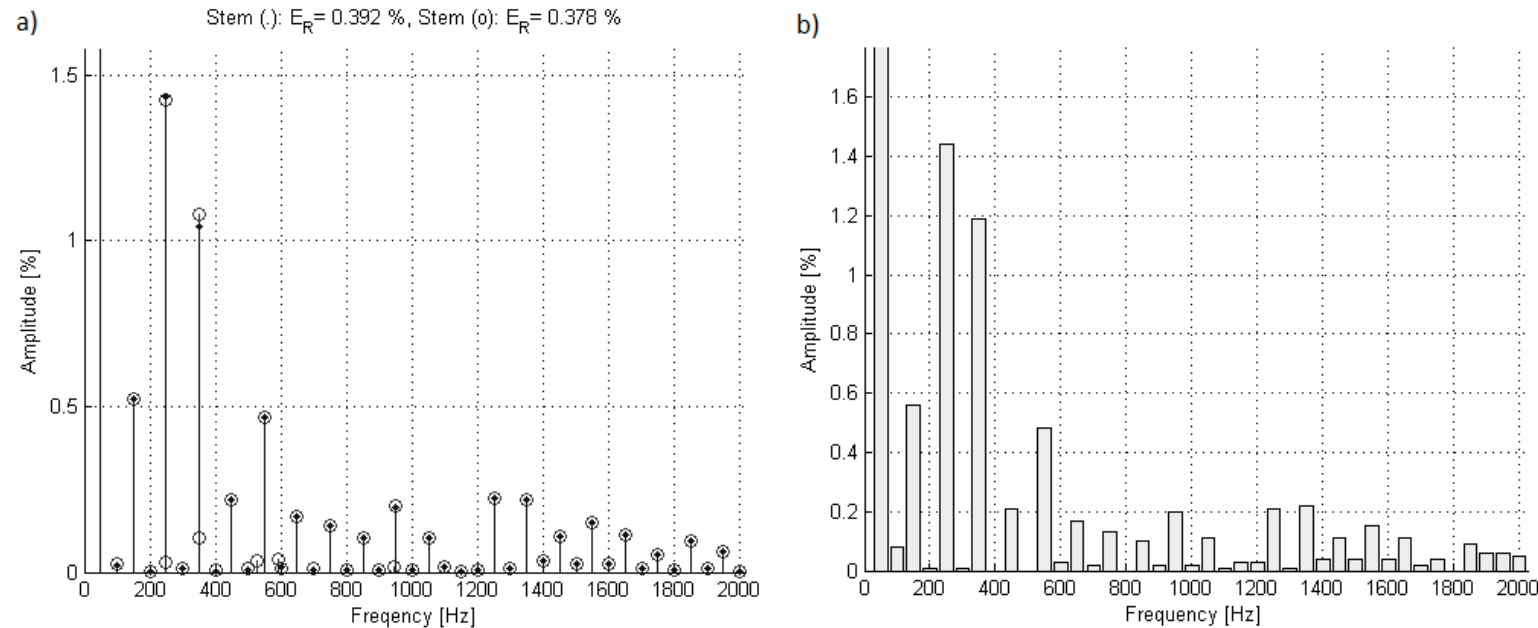

Fig. 15. Diagrams of amplitudes determined for real signal \#1, with the use of: a) modified method (points 'o') and reduced Prony method without determining interharmonics (points '.'); b) Elspec G4430.
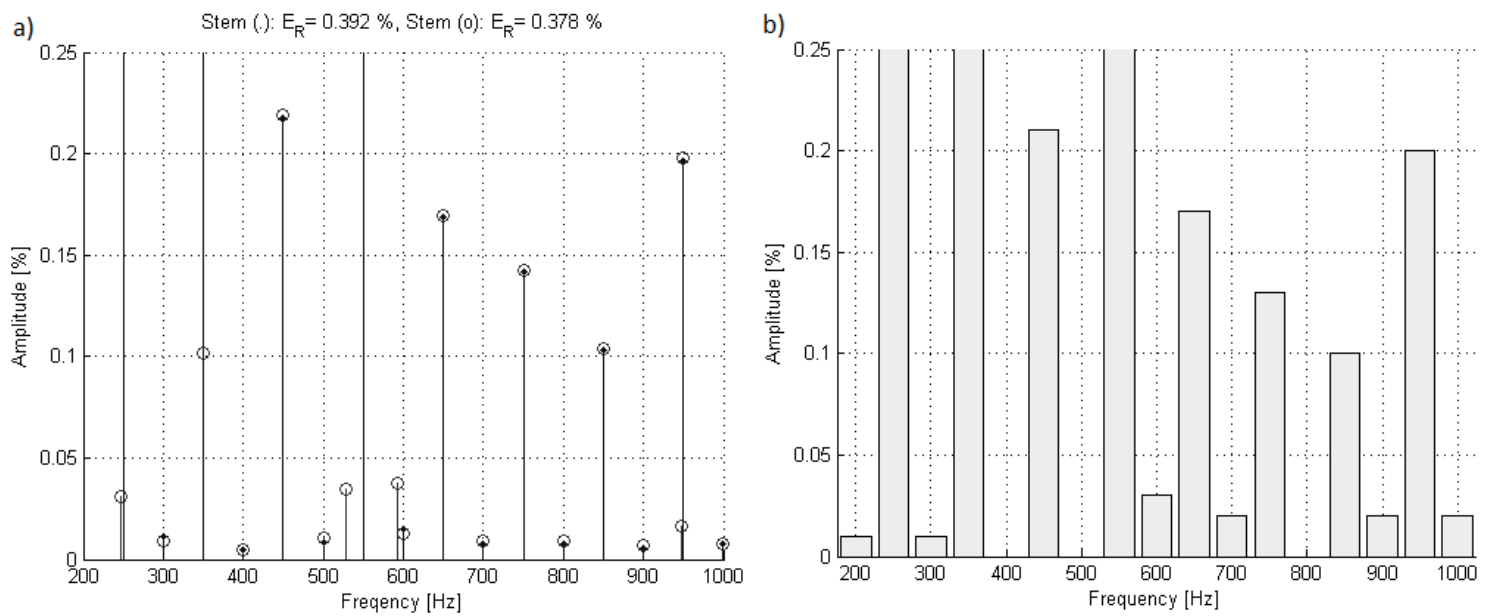

Fig. 16. Enlargement of diagrams presented in Fig. 15. 
a)

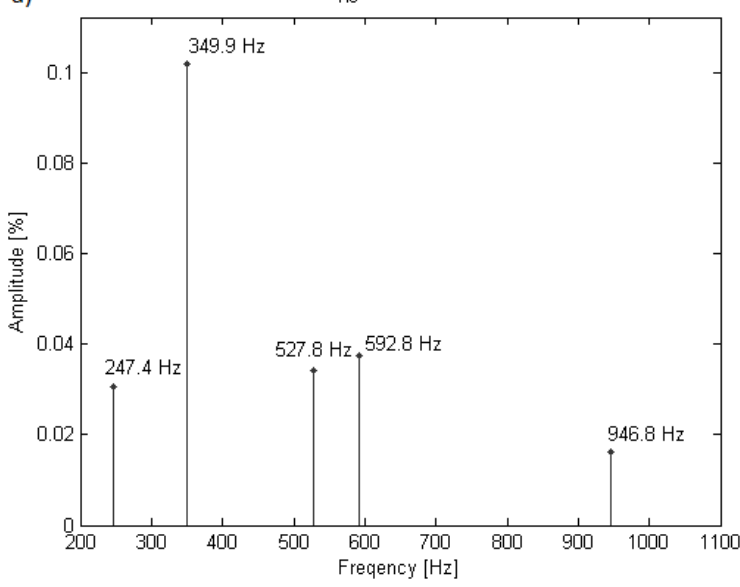

b)

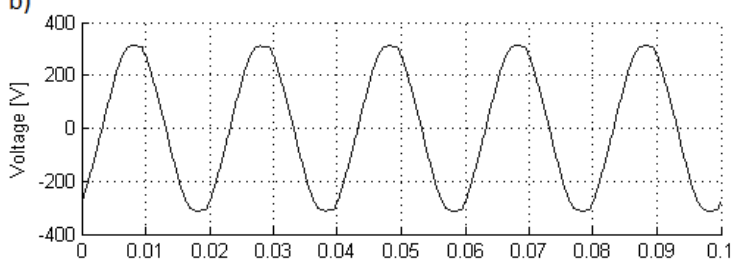

c)

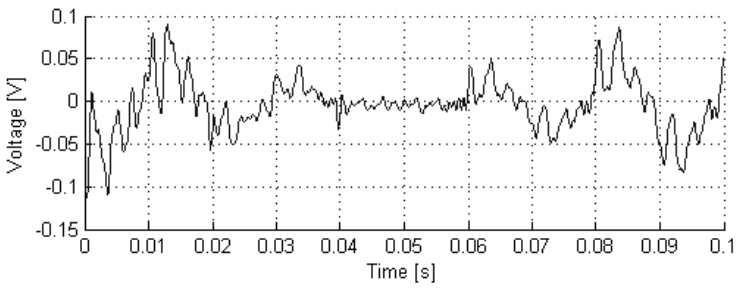

Fig. 17. Diagrams of: a) determined interharmonics; b) signal transient; c) signal reconstructed on the basis of the determined interharmonics, for real signal \#1.
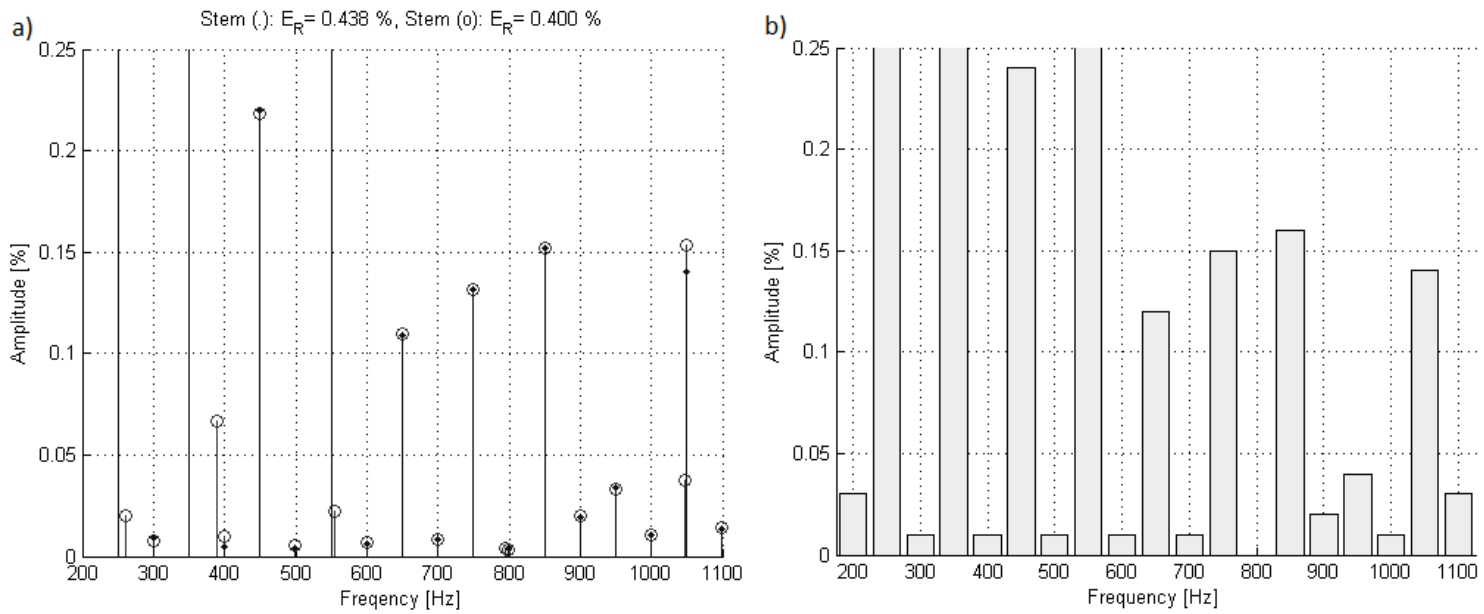

Fig. 18. Enlargement of diagrams of amplitudes determined for real signal \#2, with the use of: a) modified method (points 'o') and reduced Prony method without determining interharmonics (points '.');

b) Elspec G4430.

a)

a)

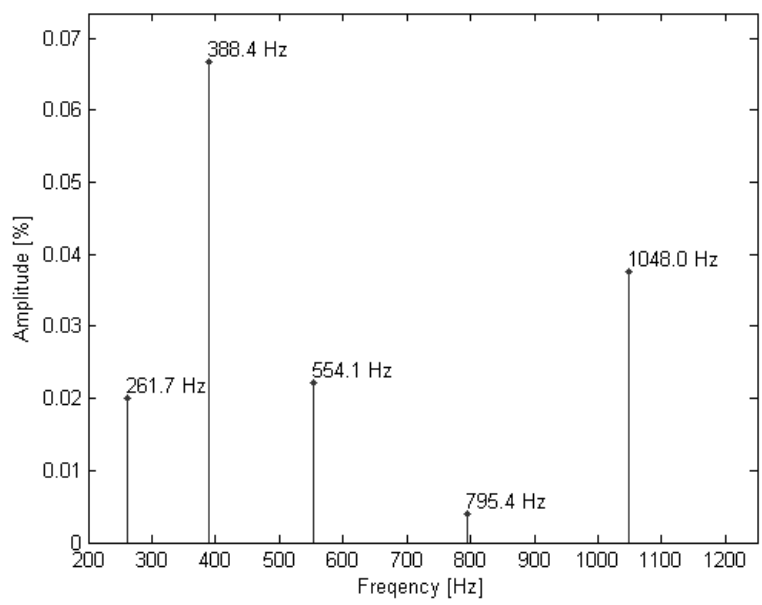

b)

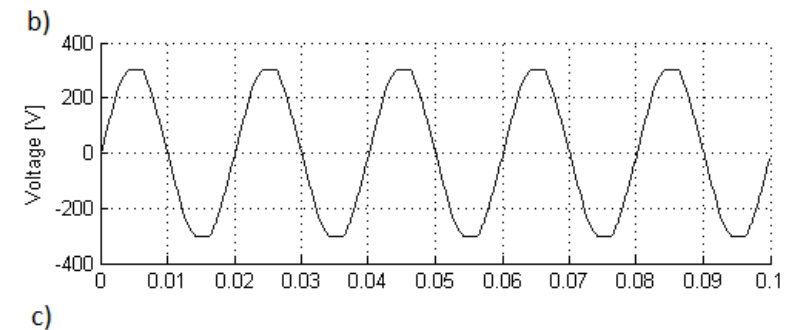

c)

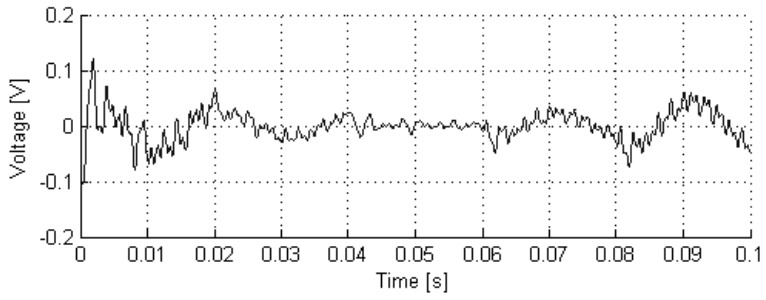

Fig. 19. Diagrams of: a) determined interharmonics; b) signal transient; c) signal reconstructed on the basis of the determined interharmonics for real signal \#2. 

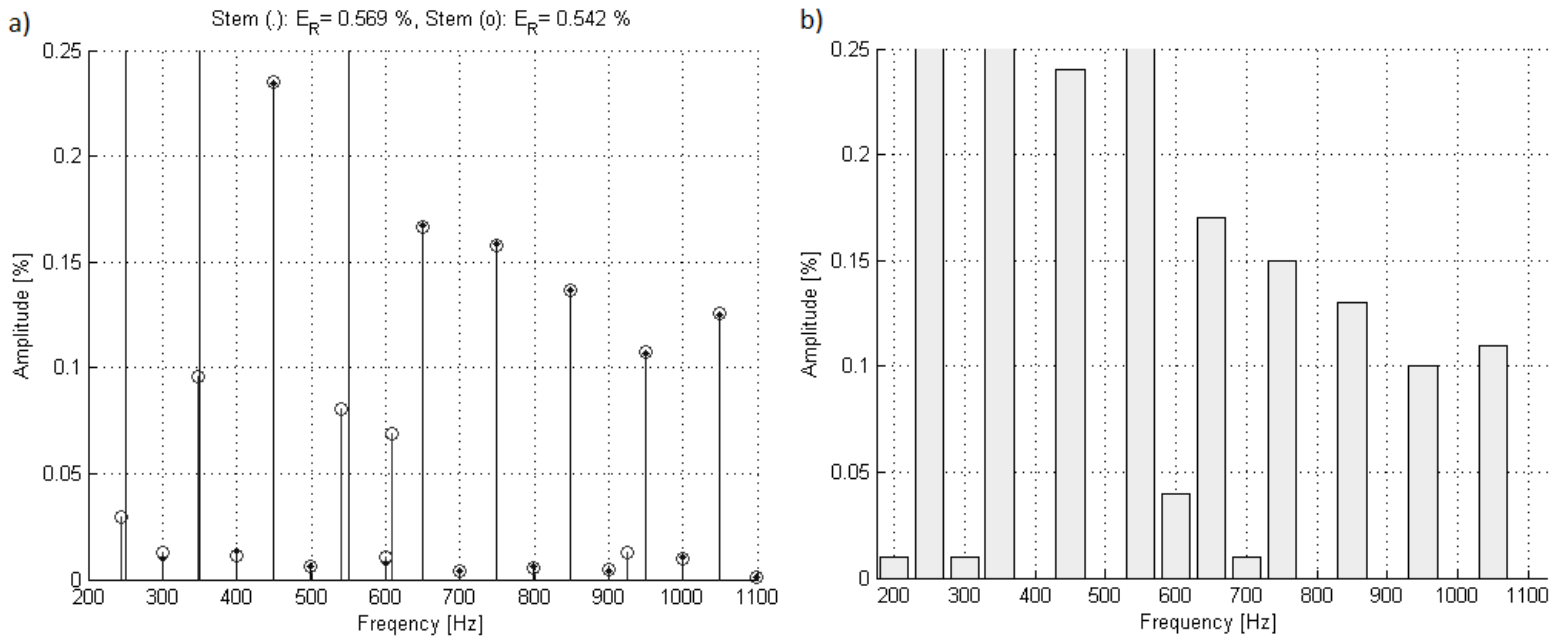

Fig. 20. Enlargement of diagrams of amplitudes determined for real signal \#3, with the use of: a) modified method (points 'o') and reduced Prony method without determining interharmonics (points '.'); b) Elspec G4430.

a)

a)

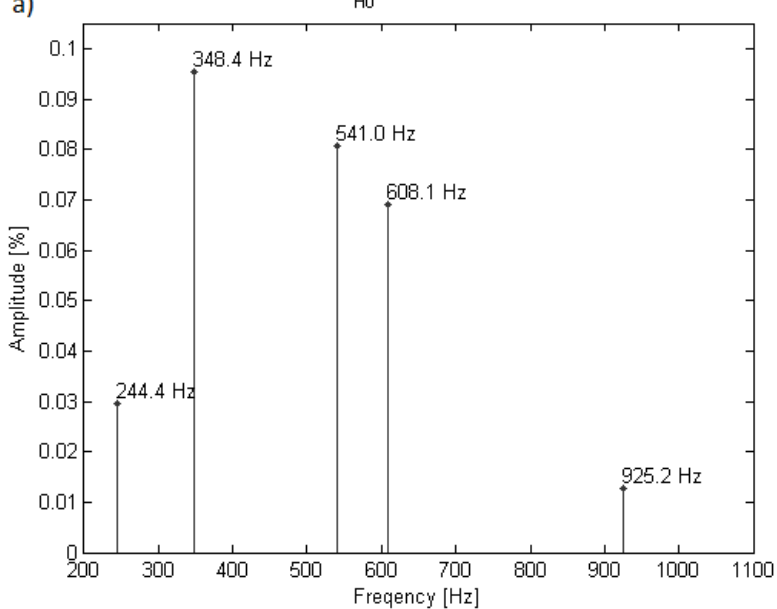

b)

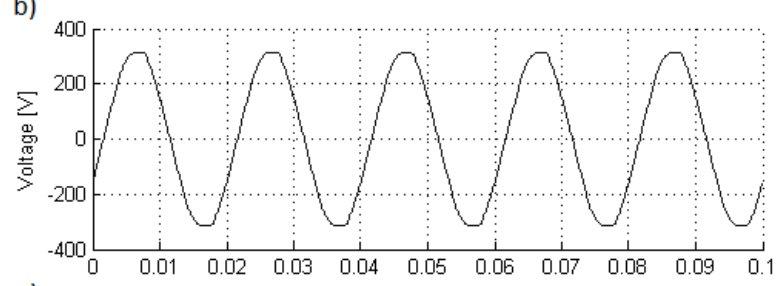

c)

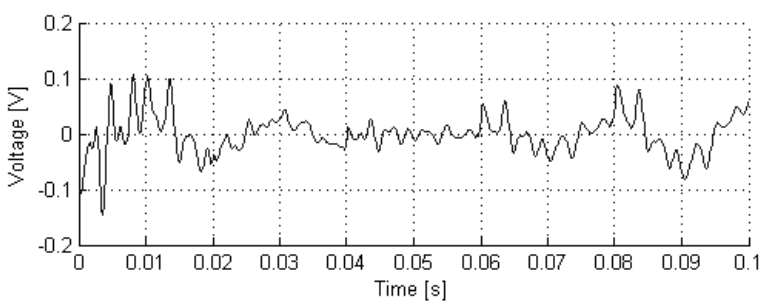

Fig. 21. Diagrams of: a) determined interharmonics; b) signal transient; c) signal reconstructed on the basis of the determined interharmonics for real signal \#3.

\section{Discussion of results}

Presented tests of the method of analyzing harmonics and interharmonics of electric power signals show that the proposed method can be used for analysis of the quality of electric power, offering new possibilities of measuring real frequencies of interharmonics. Current devices operating on the basis of a standard [7], where measurement is performed for groups of set frequency values within the tested harmonic do not offer such an option.

Fig. 7 shows the arrangement of calculated zeros of the FIR filter for basic and modified versions of the algorithm. Transient characteristics of the created FIR filters presented in Fig. 8 confirm the appropriate determination of filter parameters; components with known frequencies will be attenuated prior to Section 1 of Prony's method.

Figs. 10 and 13 present coefficients $\underline{z}_{k}$ found in Section 1 of Prony's method for unknown components. On the basis of these coefficients, analogically to Fig. 8, amplitude characteristics for relative FIR filters can be drawn - Figs. 11 and 14.

The proposed method determines interharmonics of the analyzed signals, which results in limiting reconstruction errors $E_{R}$, calculated for signals reconstructed with the use of 
interharmonics - Figs. 15, 18, 20 (Stem (o): $E_{R}$ and Stem (.): $\left.E_{R}\right)$. As compared to traditional methods, the accuracy of interharmonics frequency determination is high, yet difficult to estimate, due to its strong dependence on the "content of the analyzed signal" [13]. Comparative studies of the analyzed methods - Figs. 6-14 have shown lesser errors in determined parameters for the modified method, which made it suitable for application in the analysis of electric power quality. Considering the variable nature of the analyzed power signals, the accuracy of determining component frequencies could be at the level of $\pm 0.1 \mathrm{~Hz}$ (Fig. 12), and the amplitude at ca. $0.03 \%$. Such type of studies are covered more broadly in publication [13].

The basic method for the prepared set signals has not revealed additional interharmonics, which lead to great errors in $E_{F}$ and $E_{A}$ parameters. However, this method has shown great accuracy in revealing interharmonics for signals with a small number of harmonics, which disqualifies it in applications aiming at testing electric power quality.

The proposed modified method also reduces the computational complexity of the entire algorithm. The order of Prony's method in part I of the algorithm in which e.g. complex roots of polynomials are calculated, is reduced in order to increase the size of the FIR filter with low computational complexity. That allows to reduce the total calculation capacity. The subject will be elaborated more broadly during implementation of the algorithm hardware.

Comparison of the results obtained for real signals shows discrepancies in the values of amplitudes determined for individual harmonics by means of the proposed modified method and the G4430 device. These discrepancies can result from inaccuracies of the circuits of testing devices, but also from interharmonics with transients presented in Figs. 17, 19 and 21 (bottom right), affecting negatively the accuracy of harmonics determination in the G4430 device.

If several components appear near the harmonic, this may be a result of non-linear loads present in the electric power network and the occurrence of the modulation phenomenon. These components can be also found in the results of the Prony analyses, for example with changing frequency of a given component in a studied analysis window. The authors intend to study these phenomena in the future.

Fig. 16b, which shows results of the analysis from the Elspec device, does not show components 400 and $800 \mathrm{~Hz}$ which can be seen in Fig. 16a. Assuming class A accuracy of the Elspec device, where the harmonic amplitude error should be $5 \% U_{m}$ for $U_{m} \geq 1 \% U_{N}$ and $0.05 \% U_{N}$ for $U_{m}<1 \% U_{N}$, where $U_{m}$ is the measured voltage value and $U_{N}$ is the expected value at the input of the testing device, lack of the mentioned components may be considered an admissible measurement error of the device.

\section{Conclusions}

The method presented in the article allows to measure all parameters of harmonics, interharmonics and subharmonics of electric power signals. The method outperforms the full Prony method in that it provides faster possibilities and better stability of calculation by introducing to the algorithm information about known frequencies of sinusoids.

The described algorithm can bridge the gap between methods of analysis based on Fourier transform and full Prony methods. The method offers lesser computational complexity by permitting the use of the reduced Prony model - contrary to the full Prony method at the first calculation stage, and allowing accurate determination of component frequencies, which Fourier analyses do not provide. 
Measurement of real component frequencies for interharmonics characterized by slight differences in frequency values makes it possible to analyze power signals on a new level that is not yet available to the devices based on Fourier transform. The implemented method has great significance in the era of expanding power networks by wind power plants generating new types of interharmonics-based disturbances. The presented solution also allows to analyze the phenomena generated by voltage converters and chopper power supplies, or even by compact light sources that are becoming more and more popular in low-voltage power networks. The interharmonics observed in real signals did not reveal high amplitudes, yet the authors suppose that the possibility of detecting and analyzing them in a more accurate way should gain more importance for future diagnostics.

\section{References}

[1] Zygarlicki, J., Mroczka, J. (2011). Short time algorithm of power waveforms fundamental harmonic estimations with Prony's methods use. Metrol. Meas. Syst., 18(3), 33-38.

[2] Nam, S.R., Kang, S.H., Park, J.K. (2002). An analytic method for measuring accurate fundamental frequency components. IEEE Trans. Power Del., 17(2), 405-411.

[3] Wen, H., Teng, Z., Wang, Y. (2011). Simple Interpolated FFT Algorithm Based on Minimize Sidelobe Windows for Power-Harmonic Analysis. IEEE Trans. Power Electronics, 26(9), 2570-2579.

[4] Sachin, K.J., Singh, S.N. (2011). Harmonics estimation in emerging power system: Key issues and challenges. Electronic Power Systems Research, 81(9), 1754-1766.

[5] Borkowski, J. (2011). Minimization of maximum errors in universal approximation of the unit circle by a polygon. Metrol. Meas. Syst., 18(3), 391-402.

[6] Bracale, A., Carpinelli, G., Leonowicz, Z., Lobos, T., Rezmer, J. (2008). Measurement of IEC Groups and Subgroups using Advanced Spectrum Estimation Methods. IEEE Trans. Instr. and Meas., 57(4), 672-681.

[7] Standard PN-EN 61000-4-7:2007/A1:2011 - Testing and measurement techniques - General guide on harmonics and interharmonics measurements and instrumentation, for power supply systems and equipment connected thereto.

[8] Lobos, T., Leonowicz, Z., Rezmer, J., Schegner P. (2006). High-resolution spectrum-estimation methods for signal analysis in power systems. IEEE Trans. Instrum. Meas., 55(1), 219-225.

[9] Leonowicz, Z., Lobos, T., Rezmer, J. (2003). Advanced spectrum estimation methods for signal analysis in power electronics. IEEE Trans. Ind. Electron., 50(3), 514-519.

[10] Zhijian, Hu, Jianquang, Guo, Mei, Yu, Zhiwei, Du, Chao, Wang. (2006). The Studies on Power System Harmonic Analysis based on Extended Prony Method. 2006 International Conference on Power Systems Technology (POWERCON), Chongqing, China, 22-26.

[11] Marple, S., Lawrence, J. (1987). Digital Spectral Analysis. Prentice-Hall.

[12] Zygarlicki, J., Zygarlicka, M., Mroczka, J., Latawiec, K. (2010). A reduced Prony's method in power quality analysis - parameters selection. IEEE Transactions on Power Delivery, 25(2), 979-986.

[13] Zygarlicki, J., Zygarlicka, M., Mroczka, J. (2008). Prony's method in power quality analysis. Proc. 9th Int. Scientific Conf. Electric Power Engineering, Brno, Czech Republic, 115-119.

[14] Standard EN 50160 (2010) - Voltage characteristics of electricity supplied by public distribution networks. 